\title{
Crisis y cambio social en Chile (2010-2013): el lugar de los medios de los movimientos sociales y de los activistas digitales*
}

\author{
Raúl Rodríguez ${ }^{* *}$ \\ Patricia Peña ${ }^{* * *}$ \\ Chiara Sáez ${ }^{* * * *}$ \\ Recibido: Enero 15 - Aprobado: Marzo 15
}

\begin{abstract}
Resumen
El presente artículo analiza las catástrofes naturales (y posterior proceso de reconstrucción) del 27F de 2010 (terremoto y maremoto), el ciclo de alza de la movilización estudiantil surgido a partir de 2011, así como los distintos conflictos socio-ambientales que han estallado en distintas regiones del sur y norte de Chile desde 2010 hasta la fecha, desde la perspectiva de los medios, de los movimientos sociales y el rol de los activistas digitales en este proceso.

A partir de la sistematización de diversas fuentes propias y de fuentes secundarias, repasamos el comportamiento / uso de medios de la esfera pública convencional, medios comunitarios y la web social ante este ciclo de movilización social, e intentamos responder a la siguiente paradoja: por qué esta fase de alza en las movilizaciones caracterizada por el uso expresivo de Internet y la web social como neoesfera pública no llega a cuestionar, como parte de sus demandas, el problema económico e ideológico de la
\end{abstract}

* El artículo sistematiza una línea de trabajo en Comunicación y Ciudadanía llevada a cabo como Programa de Libertad de Expresión y Ciudadanía del Instituto de la Comunicación e Imagen de la Universidad de Chile entre 2011 y 2013. Asimismo, Raúl Rodríguez es el Director de la Radio Juan Gómez Millas y miembro de la mesa chilena de AMARC. Patricia Peña y Chiara Sáez forman parte de la Mesa de Ciudadanía y Televisión Digital.

** Periodista y magíster en Comunicación de la Universidad de Chile. Académico del Instituto de la Comunicación e Imagen - ICEI de la Universidad de Chile y Director de la Radio Comunitaria Juan Gómez Millas de esta unidad académica. Representante del capítulo chileno de la Asociación Mundial de Radios Comunitarias, AMARC. Dirección electrónica: radiojgm@uchile.cl

*** Periodista y magíster en Comunicación de la Universidad Diego Portales. Master of Science en Comunicación, Nuevos Medios y Sociedad de la London School of Economicos. Profesor Asistente y Coordinadora Académica del Diplomado en Comunicación Digital del Instituto de la Comunicación e Imagen - ICEI de la Universidad de Chile. Dirección electrónica: patipena@uchile.cl

*** Socióloga de la Universidad Católica de Chile. Doctora en Comunicación y posdoctorada del Instituto de Gobierno y Políticas Públicas de la Universidad Autónoma de Barcelona. Integrante de la Mesa de Ciudadanía y TV Digital (www.ciudadaniatv.cl) como representante del Instituto de la Comunicación e Imagen (ICEI) de la Universidad de Chile donde es Profesor Asistente desde 2011. Dirección electrónica: chiara.saez. baeza@uchile.cl 


\title{
Crisis and Social Change in Chile (2010-2013): The Place of Social Movements and Digital Activists
}

\begin{abstract}
This article is an analysis of natural disasters (and future reconstruction process) of the 27F of 2010 (earthquake and seaquake), the cycle of students protest from 2011, and several social-environmental conflicts that have occurred in several regions of southern and northern Chile since 2010 to date from the point of view of media, social movements, and the role of digital activists in this process.
\end{abstract}

From systematization of several own sources and secondary sources, a review of the behavior/use of conventional public media, community media, and social web before this social mobilization cycle has been made trying to respond to the following question: why does this cycle - characterized by the expressive use of the Internet and the social web as public neosphere - not question - as part of its demands - the economic and ideological problem of concentration of media system in Chile in a period in which relevant debates about this topic are simultaneously generated? Just as the case of the Digital TV law or the community radio law.

In our opinion, in order to explain this apparent contradiction, answers should be found out of a media-associated approach which establishes at least three factors: citizen participation, communicational citizenship, and techno-utopian thinking.

Key words: Social movements; communication; citizenship; digital activism; public sphere.

concentración del sistema mediático en Chile, en un período en el cual simultáneamente se generan debates relevantes al respecto, como es el caso de ley de TV digital o la ley de radios comunitarias.

Para poder explicar esta aparente contradicción consideramos que las respuestas deben buscarse por fuera de un enfoque mediocéntrico, que problematice, al menos, tres factores: participación ciudadana, ciudadanía comunicativa y pensamiento tecnoutópico.

Palabras clave: movimientos sociales, comunicación, ciudadanía, activismo digital, esfera pública 


\section{Introducción}

El terremoto y maremoto del 27 de septiembre de 2010, como hito simbólico, y la vuelta de la derecha al gobierno, a las semanas siguientes, como hito político, se constituyen en la inflexión que inaugura un cambio de época en las movilizaciones y formas de articulación social en Chile.

Según un estudio publicado por el Instituto Nacional de Derechos Humanos (INDH, 2012), en Chile se registran 97 conflictos socioambientales a partir del año 2010, entendiendo bajo este concepto

Disputas entre diversos actores [...] manifestadas públicamente y que expresan divergencias de opiniones, posiciones, intereses y planteamientos de demandas por la afectación (o potencial afectación) de derechos humanos, derivada del acceso y uso de los recursos naturales, así como por los impactos ambientales de las actividades económicas (5).

El origen de este proceso puede remontarse, según Cuenca (2012: 7), a los acuerdos adoptados en Río 92 y que han resultado en "políticas que permitieron la privatización de la naturaleza y que han llevado al país a un estado de conflictividad socioambiental sin precedentes, cooptando a gran parte de los medios de comunicación, las autoridades y la academia". Estos conflictos que emergen o cristalizan a partir de 2010 dan lugar a diversas movilizaciones de carácter territorial y medioambiental a lo largo del país (Punta de Choros, HidroAysén, Punta Arenas).

Sin embargo, va a ser la campaña contra el proyecto hidroeléctrico HidroAysén la que va a tener mayor impacto en términos políticos, dada su masividad (más de 70 mil personas en Santiago y otras 20 marchas en todo Chile) y la conexión que se produjo entre esta demanda social y la de los estudiantes, hasta llegar a cuestionar las estructuras más elementales del actual orden político. Según Segura (2012):

[.. . .] muchos de los líderes universitarios protagonistas de ese permanente 'Mayo del 68' en que se convirtió Chile durante todo 2011 habían sido partícipes de las manifestaciones previas contra las represas en la Patagonia [... Las protestas contra HidroAysén fueron también el inicio de un proceso que ha permitido cuajar el cuestionamiento al modelo socioeconómico vigente, incrustado en la Constitución del 80 (29).

El año 2011 abre un nuevo ciclo de movilizaciones estudiantiles, convocadas tanto por estudiantes universitarios como por secundarios. Su objetivo es el rechazo al fuerte carácter privado y comercial del sistema educacional chileno, que se origina durante la dictadura de Pinochet, la que deja al Estado como regulador antes que como garante de la educación al delegar gran parte de la enseñanza al sector privado. Si bien este ciclo de movilización es considerado uno de los más importantes de la posdictadura, está conectado con otros ciclos de movilización estudiantil previos que han sido permanentes desde la década de los 90, y tiene como antecedente más cercano la denominada Revolución Pingüina de 2006. En este mismo sentido, puede señalarse que este ciclo de movilizaciones es el mismo que perdura hasta hoy. 
Si bien durante 2012 el movimiento estudiantil se mantiene en una fase de baja intensidad, explotan nuevos conflictos relacionados con medio ambiente y energía a lo largo del país (Aysén, Pelequén, Freirina, Petorca, Huasco) y tanto los medios comunitarios como las redes sociales y diversas formas de activismo digital mantienen informados a los agentes movilizados, aunque en una lógica atomizada.

Dado este contexto, nuestra pregunta de investigación es: ¿por qué estas movilizaciones sociales y ciudadanas, caracterizadas por la extensión de un uso expresivo de Internet y la web social como neoesfera pública, no superan una lógica de marketing y, por lo tanto, no problematizan como parte de sus demandas el sentido transformador de la comunicación ni tampoco problematizan la concentración económica y la "homogeneidad ideológica" (Sunkel y Geoffroy, 2001: 106) que caracterizan el sistema mediático en Chile?

Nuestras hipótesis, en tanto, se relacionan con lo que observamos como una perspectiva utópica en torno a las tecnologías digitales por parte de diversos agentes de la movilización social, la cual no promueve una apropiación social de las tecnologías en un sentido profundo y que puede comprobarse comparando el uso de la web social con otras formas de comunicación en el contexto de las movilizaciones.

\section{Marco teórico: movimientos sociales, medios de los movimientos sociales y activismo digital}

Diversos autores han manifestado su preocupación sobre el "persistente divorcio" (Cammaerts, 2012: 118) entre, por una parte, la investigación de los movimientos sociales por parte de historiadores, politólogos y sociólogos, y por otra, la investigación sobre los usos, apropiaciones y conformación de los proyectos comunicativos propios de los movimientos sociales, así como las relaciones de estos con los medios convencionales.

En esta articulación los medios con frecuencia "son definidos simplemente como canales tecnológicos de mensajes, antes que como las complejas instituciones sociotécnicas que actualmente son" (Downing, 2008: 41). A pesar de que como señala Rovira (2012) "la comunicación es una de las actividades definitorias de cualquier movimiento social" (1).

Para Tilly y Wood (2009) los movimientos sociales se caracterizan por la síntesis de tres elementos: revindicaciones (campañas), un repertorio de formas combinadas de acción política y manifestaciones públicas y concertadas de ello (demostraciones). El movimiento social no se constituye por una sola de estas características, sino por la interacción / combinación entre ellas. De manera que la acción política popular deviene movimiento social en aquellos

[...] lugares y momentos en los que la gente que planteó reivindicaciones colectivas contra las autoridades formó con frecuencia asociaciones con un fin específico o dio un nombre a las coaliciones surgidas, celebró reuniones públicas, trasladó sus programas a los medios de comunicación a su disposición, organizó marchas, reuniones o manifestaciones y llevó a cabo, por medio de estas actividades, demostraciones públicas concertadas de valor, unidad, número y compromiso (Tilly y Wood, 2009: 69). 
Para Tarrow (2004) el surgimiento de los movimientos sociales responde a una estructura de oportunidades políticas; vale decir que los movimientos sociales se dan cuando se producen cambios en la estructura de las oportunidades y restricciones políticas que crean los incentivos para nuevas acciones. No obstante, utiliza la categoría más genérica de acción colectiva contenciosa, para abarcar movimientos sociales, ciclos de protesta y revoluciones. Este concepto es pertinente dado el carácter descentralizado en el cual se produce la acción colectiva contenciosa actualmente, y que de algún modo lleva a una insatisfacción con el concepto más fijo de movimiento social.

Por esta razón, más que entrar a debatir si nuestros casos de análisis (movilizaciones posterremoto, movimiento estudiantil y movimientos socio-ambientales) se ajustan completamente a las definiciones académicas de movimientos sociales, sí nos parece adecuado establecer como punto de partida la idea de que el año 2010 se inicia en Chile un nuevo ciclo de acción colectiva -cuya traducción en forma de movimiento social se encuentra aún en proceso-, entendiendo bajo ese concepto

Una fase de intensificación de los conflictos y la confrontación en el sistema social, que incluye una rápida difusión de la acción colectiva de los sectores más movilizados a los menos movilizados, un ritmo de innovación acelerado en las formas de confrontación, marcos nuevos o transformados para la acción colectiva, una combinación de participación organizada y no organizada y unas secuencias de interacción intensificada entre disidentes y autoridades (Tarrow, 2004: 202-203)

Para comprender la relación entre comunicación y movimientos sociales conviene partir citando el trabajo de Rucht (2004), quien analiza los movimientos sociales en Estados Unidos (los nuevos movimientos sociales de los 60 como los actuales movimientos globales) ante la falta de resonancia de sus actividades y demandas en los medios de comunicación distinguiendo cuatro tipo de reacciones fundamentales, a las que denomina "cuatro A": abstención, ataque, adaptación y alternativas.

La abstención consiste en abandonar los intentos de influenciar a los medios y resulta de las experiencias negativas con los medios establecidos. El ataque consiste de una crítica explícita e incluso a veces de acción violenta hacia los medios. La adaptación significa la aceptación o explotación de las reglas y criterios de los media, con el propósito de influir positivamente las coberturas mediáticas, siendo que los movimientos más reformistas tienden a aplicar esta estrategia. Por último, la alternativa es el intento de los movimientos sociales de crear sus propios medios o espacios públicos de comunicación independiente. Según el autor, solo esta última estrategia refuerza en el largo plazo la dimensión comunicativa de los movimientos sociales a favor de las transformaciones sociales.

El concepto de medios de los movimientos sociales (Downing, 2010; 2011a), si bien mantiene el acento en el carácter contrahegemónico de la comunicación alternativa, pone a estos medios en relación con los procesos de movilización y acción colectiva: "es común que aunque los movimientos sociales estén en declive, el medio mantenga la llama viva en torno a un determinado tema durante el periodo entre la implicación activista actual y la futura. Por eso estos medios no son solo productos de los movimientos sociales sino 
que también los nutren, incluso cuando se encuentran en una de sus fases 'subterráneas' (Downing, 2010)".

En la articulación entre comunicación y movimientos sociales, Downing también acuña el concepto de Medios efímeros, que corresponden a expresiones de la comunicación alternativa de carácter más performativo, que incluso tendrían la propiedad de alojarse más fácilmente en la memoria, precisamente a partir de su concentración en un solo instante, su "one-shot concentraded pungency" (Downing, 2001: 102). Incluyen "desde el teatro callejero y los murales hasta la danza y el canto [... ] y no meramente el uso radical de las tecnologías detrás de la radio, el vídeo, la prensa e Internet (Downing, 2011b: 414)".

Sin embargo, no nos parece adecuado establecer una relación unívoca entre medios de los movimientos sociales y activismo digital: no todo el activismo digital se articula en forma de comunicación de los movimientos sociales. A veces, solo corresponde a instancias de autoexpresión.

La web 2.0 (o web social) se refiere al desarrollo de Internet que ha amplificado las posibilidades de expresión y difusión, permitiendo una ciudadanía activa que puede participar colaborativamente en la construcción colectiva de un relato sobre un determinado hecho de alto impacto. Es lo que señalan autores como Lara o Castells al referirse a los procesos de movilización social que a partir de 2011 han recorrido diversos países. La primera pone el énfasis en la politización de la experiencia personal: "el éxito de Twitter demuestra que 'lo personal es político' ya que, en cierta forma, con nuestros fragmentos y huellas digitales, estamos dejando los relatos con los que se construirá nuestra historia social en un futuro" (Lara, 2012: 29), mientras el segundo sostiene que en los últimos años el cambio fundamental en el mundo de las comunicaciones ha sido el nacimiento de la llamada autocomunicación de masas:

Es comunicación de masas porque procesa mensajes de muchos para muchos y potencialmente puede llegar a muchos receptores y conectarse a incontables redes que transmiten información digitalizada en un barrio o por todo el mundo. Es autocomunicación porque el emisor decide el mensaje de forma autónoma, designa a los posibles receptores y selecciona los mensajes de las redes digitales que quiere recuperar (Castells, 2012: 24)

Desde los usos autoexpresivos de la comunicación digital que "inician en la red" movimientos "en gran medida espontáneos", es difícil plantearse las relaciones de continuidad entre las tecnologías digitales y el activismo comunicacional de movimientos y organizaciones. Por el contrario, seguimos en este punto el planteamiento de Tilly y Wood (2009) en el sentido de evitar el determinismo tecnológico, identificando, asimismo, qué es lo nuevo, lo distintivo y lo recursivo en el activismo digital, y de qué modo se conecta con la historia larga del activismo social:

Ni Facebook ni otras redes con una penetración menor poseen la capacidad para llevar a cabo el trabajo político sostenido en nombre de sus programas que sí hemos visto en situaciones de siglos pasados a la hora de consolidar el repertorio del movimiento social [...] nada de todo esto da voz a la gente corriente a la hora de tomar decisiones (238). 


\section{Metodología}

El presente artículo sistematiza, desde una perspectiva comunicacional, los hallazgos provenientes de distintas fuentes (entrevistas, observación participante y sistematización de estudios realizados por terceros) sobre los tres casos de análisis:

El terremoto + tsunami del 27 de febrero de 2010 y el proceso de reconstrucción

La ola de movilización estudiantil que se levanta a partir de 2011

Los diversos conflictos socioambientales que se generan a partir de 2010 en distintas zonas del país

Las fuentes primarias incluidas son las siguientes':

Entrevistas en profundidad a coordinadores y líderes de TV para Chile y Cadena Radial, dos proyectos de comunicación desarrollados por estudiantes universitarios en el contexto del movimiento estudiantil de 2011.

Entrevistas en profundidad a gestores (hombres y mujeres) de proyectos de radios comunitarias en zonas afectadas por el terremoto del 27 de febrero de 2010. En este caso, el levantamiento de datos se llevó a cabo en dos fases diferentes. Durante marzo de 2010 y de marzo a noviembre de 2011. En el segundo caso el estudio estuvo más centrado en el proceso de reconstrucción y el rol de las radialistas comunitarias mujeres.

Como experiencias de observación participante utilizadas para arribar a los resultados de esta investigación, se incluyeron las siguientes fuentes:

Seguimiento de las estrategias de voluntariado digital en el contexto posterremoto, por medio de la participación, y registro sonoro de 3 foros realizados en el tema durante 2010: Encuentro Global Voices (marzo 2010 en la Biblioteca de Santiago), Conferencia de Jenna Dawn -la Directora Mundial de Comunicaciones de Twitter- en Chile (octubre 2010 en la Universidad Diego Portales) y la Conferencia de Personal Democracy Forum (noviembre 2010 en el edificio de la Telefónica).

Asistencia a las marchas por la educación de 2011 (6 en total), haciendo registro visual y fotográfico de las experiencias de auto-expresión observadas.

Acompañamiento presencial de la primera emisión de TV para Chile: "EnComunicación: 24 horas por la educación" (21 y 22 de julio de 2011) y de la segunda emisión "1800 minutos por la educación" (30 y 31 de agosto de 2011).

Asistencia a talleres (3) del proyecto Levantemos Ciudadanía de la Asociación Chilena de ONG, ACCIONAG.CL2, entre 2011 y 2012, en el contexto del proceso de reconstrucción pos-27F.

1 Para mayor detalle sobre protocolo y muestra de cada fuente, ver Anexos.

2 http://accionag.cl/noticias/levantemos-ciudadania-organizaciones-de-la-sociedad-civil-cumplen-rol-protagonico-en-la-reconstruccion/ 
Asistencia taller (1) del proyecto Reconstruyamos Ciudadanía de la ONG Educación y Comunicación, ECO en 2012, en el contexto del proceso de reconstrucción pos-27F.

Por último, debe considerarse como parte de los insumos para este artículo la investigación participativa desarrollada por el equipo investigador, a saber:

Dirección del programa radial Noticiero Ciudadano (desde marzo 2010 hasta la fecha) que se emite a partir del terremoto del 27 de febrero de 2010 y en el cual se enlaza y redifunde la información de diversas radios comunitarias, ciudadanas y alternativas del país. Los principales temas de cobertura del Noticiero ciudadano han sido educación y medio ambiente, seguidos de reconstrucción (sobre todo en el momento inmediatamente posterior al 27F), derechos humanos, pueblos indígenas y relaciones laborales. Para el caso de los conflictos socio ambientales del período, las comunidades afectadas han participado del noticiero en tanto reporteros comunitarios o ciudadanos, así como en calidad de testigos y afectados. Todas las transmisiones del Noticiero Ciudadano están digitalizadas, por lo que han sido utilizadas como material de consulta.

Dirección general de la primera emisión de la Cadena Radial: "1800 minutos por la educación" (9 y 10 de agosto de 2011).

Participación en la Mesa de Ciudadanía y Televisión Digital (desde 2011 hasta la fecha), expresada en la producción de documentos académicos, columnas de opinión, entrevistas para medios en distintos soportes, diálogo con parlamentarios y personeros de gobierno, acciones de difusión pública, reuniones de trabajo con representantes de los productores audiovisuales, las televisiones comunitarias, organizaciones de trabajadores de la industria televisiva, entre otras.

Entre las fuentes secundarias se consideran diversas publicaciones y estudios focalizados en distintos aspectos de cada uno de los casos de análisis. Entre ellos, el estudio de Alejandro Barros (Barros, 2010) sobre el impacto del terremoto en las infraestructuras; el estudio del Consejo Nacional de TV (CNTV, 2010) sobre la cobertura del terremoto en la televisión, por medio un análisis de contenido y una encuesta; tesis recientes de pregrado y posgrado sobre comunicación y conflictos socioambientales (Olave, 2012; Moscoso y Munjin, 2013); diversas fuentes periodísticas que documentan el monitoreo como la criminalización de la movilización social vinculada al movimiento estudiantil y los conflictos socioambientales.

A partir del marco teórico y la información sistematizada se han generado las siguientes categorías que permiten analizar comparativamente los tres casos seleccionados:

I. Infraestructuras. Nos referimos con este nombre a las redes de telecomunicaciones, que a menudo son consideradas solo como un dato de la comunicación, cuando en realidad se encuentran en permanente amenaza de diversas formas de control y, por lo tanto, no son "espacios de autonomía en gran medida fuera del control de gobiernos y corporaciones" (Castells, 2012: 20). Por el contrario, "todos estos medios de comunicación tienen la ventaja, a ojos del pueblo, de que es difícil que caigan bajo el control gubernamental. 
No obstante, los Gobiernos se están adaptando rápidamente a las nuevas tecnologías" (Tilly y Wood, op. Cit. 193)

II. Medios convencionales. Rovira (2012) advierte que los movimientos sociales no controlan su propia imagen pues, aunque elaboran estrategias de cómo van a manifestarse, están sujetos a la voluntad y la agenda de los grandes medios. Por ello, la dependencia que se establece entre medios y movimientos sociales es conflictiva, en una cierta lucha de estos últimos por su legitimación y donde los medios pueden ser gestores o intermediarios entre la sociedad y el Gobierno. Así, si los movimientos sociales no están presentes en la opinión pública, es como si no existiesen para las autoridades o para aquellos decisores de las demandas de quienes protestan.

III. Medios alternativos. Llamados también populares, comunitarios y ciudadanos; en esta categoría agrupamos a los medios de comunicación que se da a sí misma la sociedad civil movilizada: formas expresivas de la esfera pública subalterna, que ocurren en los márgenes de la esfera pública convencional (aunque no autónomos de ella), conformando un circuito cultural y comunicacional alternativo al oficial, pero interdependiente con él. Se caracterizan por su gestión participativa, no-lucrativa, y su alteración de las lógicas profesionalizantes de acceso a la emisión.

IV. Medios efímeros: remite a las formas comunicativas de carácter performativo, que incluye "una enorme gama de actividades: desde el teatro callejero y los murales hasta la danza y el canto [...] y no meramente el uso radical de las tecnologías detrás de la radio, el video, la prensa e internet (Downing, 2011a: 414)

V. Web Social. Aunque la opción de comunidades de personas interactuando entre ellas es una posibilidad que ha estado contenida desde el origen de la Internet (web 1.0), es con la web social (o web 2.0) cuando las posibilidades de expresión y difusión se amplifican. Este concepto remite a las aplicaciones que facilitan el intercambio de información y diversos tipos de contenido multimedia, que tienen características de interoperabilidad, poseen un diseño centrado en el usuario/la usuaria y promueven la colaboración (O’Reilly, 2007).

\section{Resultados}

\section{a) Terremoto y reconstrucción}

Infraestructuras. Tras el desastre natural del 27F "quedó de manifiesto la criticidad de la infraestructura de comunicación y telecomunicaciones del país" (Barros, 2010: 136): sistemas de alertas, comunicaciones telefónicas (fijas y móviles), así como el acceso a servicios de Internet se vieron seriamente colapsados.

Medios convencionales. En este ámbito la televisión jugó un rol ambivalente durante la primera semana: fue "orientadora e informativa" (CNTV, 2010: 30), pero al mismo tiempo desarrolló un enfoque espectacular y emotivo para acercarse el desastre, en que "el televidente pasa a ser un mero espectador y la información un puro espectáculo (Souza y Martínez, 2011: 75). Por ello, la televisión despertó críticas de parte de las audiencias a través de denuncias ciudadanas, y motivó un dictamen del Consejo de Ética de los medios, con el propósito de "proponer 
criterios que aseguren a los medios una adecuada actitud profesional en situaciones de catástrofe, lo que contribuye a resguardar la calidad de la información" (CEM, 2010: 1). Esta deuda de la televisión espectacularizante con un público más activo es la que, pensamos, vino a cubrir la web social como espacio de construcción de discursos colectivos

Medios alternativos. No obstante su reacción con cierto retraso debido a daños en sus equipos o dependencias, las radios comunitarias se convierten en mediadoras entre las comunidades afectadas y las autoridades locales, regionales y nacionales. Al generar un vínculo más próximo con los auditores, promueven la asociatividad entre los actores y establecen canales para resolver sus demandas más urgentes. El catastro del sector realizado en 2010 tras el terremoto permite destacar el rol y el efecto que tiene la comunicación directa entre las personas en situación de aislamiento, así como la labor informativa de los primeros días que consistió en contactos con las autoridades y la prestación de servicios a la comunidad.

Pasados cuatro días después del terremoto, las radios comunitarias logran consolidar un programa en red desde la radio Juan Gómez Millas (radio online del campus del mismo nombre de la Universidad de Chile, gestionada por estudiantes, funcionarios y académicos del Instituto de la Comunicación e Imagen) llamado Noticiero Ciudadano, el cual conecta a varias radios comunitarias del país que entregan y distribuyen información en red. El Noticiero es el único proyecto comunicacional que surge y logra consolidarse tras el 27F, al punto que sigue transmitiéndose hasta la fecha en que redactamos este artículo.

En la categoría de medios efímeros, podemos citar las distintas iniciativas expresivas del denominado Movimiento Nacional por una Reconstrucción Justa, que incluyen marchas, elaboración de carteles, consignas, votaciones, conciertos, entre otras, por parte de las diversas organizaciones que la componen y que abarcan las regiones del país más afectadas por el desastre natural.

En el caso de los usos de la web social, detectamos tres acercamientos diferenciados; autoexpresión, iniciativas nacionales e internacionales de voluntariado online, e iniciativas territoriales de ONG nacionales.

Autoexpresión. Por primera vez en Chile la web (principalmente a través de redes sociales como Twitter, Facebook y Youtube) se convierte en plataforma para la difusión de relatos testimoniales de un desastre natural de gran magnitud (en el caso del maremoto, incluso antes de que las autoridades lo confirmaran). En febrero de 2010 había 100 mil usuarios chilenos en la red social de "microblogging", twitter. Luego del terremoto, esta cifra aumentó en $190 \%$ (The Lab YER, 2010). Posteriormente, en su visita a Chile, Jenna Dawn señaló que este porcentaje se incrementó en 500 \% en los tres meses siguientes y que se enviaron 2.392.839 "tweets" asociados con el hashtag \#terremotochile (América Economía, 2011).

Voluntariado online. Al igual que en el caso de terremotos como el de Haití y Japón, surgieron rápidamente iniciativas institucionales internacionales de voluntariado a través de la web social en contextos de crisis, como es el caso de Ushahidi y de ciertas aplicaciones realizadas desde Google, así como proyectos de carácter nacional, tales como Digitales por Chile, Chile 
Ayuda y Desafío Levantemos Chile. Se crearon blogs y sitios web para canalizar ayuda material y monetaria, no necesariamente coordinadas con la ayuda entregada por el Gobierno central. Sin embargo, no fructificaron en el tiempo: tienen poca acogida entre las comunidades y no consiguen convertirse en referentes de apoyo en las labores de ayuda y posterior reconstrucción.

Las ONG y el trabajo de base. Varias ONG nacionales se focalizaron en el acompañamiento de las comunidades afectadas con el objetivo de fortalecer sus propias capacidades de contraloría social en el proceso de reconstrucción, valiéndose para ello del uso de herramientas y plataformas online, como fue el caso de Reconstruye, Construyamos Ciudadanía y Levantemos Ciudadanía. Sin embargo, un punto a analizar críticamente es la incapacidad de estas iniciativas para complementar o fortalecer a las comunidades afectadas, desarrollando estrategias de capacitación, transferencia y acompañamiento en el uso y apropiación estratégica de las TIC, especialmente a través del acceso a teléfonos móviles o de Internet en lugares de acceso público

A modo de síntesis, se observa por un lado una paradójica sinergia entre la autoexpresión de individuos atomizados y "el medio más unilateral" (Castells, 1996) de todos: la TV abierta. Basta ver, por ejemplo, las posibilidades que generaron ciertos programas, para que al mismo tiempo de su emisión pudieran verse los mensajes que, vía Twitter, iba enviando el público. Por otro lado, las experiencias con fuerte base online de voluntariado y de cooperación (ONG), al ser realizadas fuera de los espacios concretos o sin incorporar adecuadamente las necesidades de los lugares críticos, no lograron mantenerse. De modo que la comunicación con las comunidades afectadas operó siempre de manera vertical, tanto en el caso de la televisión como en el caso de la mayoría de las experiencias de soporte y apoyo institucional que recurrieron a la web social, con ciertos espacios muy delimitados para la visibilidad masiva de las formas individuales online de expresión del desastre. En este contexto, solo las radios comunitarias y ciudadanas parecieron encontrar un espacio para formas de trabajo horizontal y cooperativo, con capacidad de perdurabilidad en el tiempo.

\section{b) Movimiento estudiantil}

Infraestructuras. Lo más relevante sobre este punto se relaciona con la polémica que generó el anuncio de la externalidad de un servicio de monitoreo de redes sociales por parte del Gobierno del presidente Piñera a mitad de 2011, cuando el movimiento estudiantil estaba en pleno auge. Si bien el Gobierno sostuvo todo el tiempo que era un seguimiento de tendencias -similares a los que se realizan con respecto a la prensa escrita o la televisión- e hizo pública una versión del primer informe de monitoreo (Gobierno de Chile, 2011), en la práctica el monitoreo también incluyó el seguimiento de cuentas personales en twitter y facebook, cuya consecuencia más vistosa fue la denuncia de un periodista radial, quien recibió una llamada del Ministerio del Interior para retractarse de un comentario irónico contra el Ministro de turno, a propósito de las movilizaciones estudiantiles3. El debate suscitado en torno a la libertad de expresión y la protección de datos en twitter provocó el anuncio público del fin del monitoreo de redes sociales unos meses después.

3 https://twitter.com/JuvenalRivera/status/88087163723464704 
Medios convencionales. Hubo distintos hitos a lo largo de las movilizaciones en que los estudiantes acusaron la tendenciosidad contra el movimiento estudiantil por parte de los canales de televisión, principalmente en los programas informativos. Dos de los casos más recordados: i) el canal público usando en diversas ocasiones la imagen de un encapuchado para introducir noticias sobre las marchas, incluso cuando la información no contenía referencia a hechos de violencia o desorden público, hecho denunciado ante el Colegio de Periodistas y el Consejo Nacional de Televisión (Cuello, 2011); ii) un reportaje realizado por el canal 13 (Universidad Católica-Luksic) llamado La otra cara de las marchas y que fue objeto de una denuncia de la Asociación Chilena de ONG ante el Consejo Nacional de TV, acusando una "criminalización de los movimientos sociales" (ACCIÓN, 2011). También se denunció la supuesta censura del despliegue de una bandera gigante del movimiento por la educación en los partidos de Chile en la Copa América del 2011 y que ni Canal 13 ni TVN mostraron (Prensa.cl, 2011). Hubo, asimismo, casos de asedio a los medios escritos representantes del duopolio mediático (El Ciudadano, 2011) o la entrada al set de programas en vivo por parte de estudiantes reivindicando el movimiento estudiantil, como fue el del Noticiero del canal UCV (El Mostrador, 2011) y de CHV (Emol, 2011) y el Programa Caiga Quien Caiga en Mega (Radio Bíobío, 201la). Por último, funcionarios del Ministerio de Educación denunciaron acuerdo de Mineduc con TVN y Canal 13 para mostrar desórdenes dentro de sus instalaciones (Radio Bíobío, 2011b). A diferencia de los otros dos casos analizados, en el caso del movimiento estudiantil la actitud crítica activa contra el tratamiento del tema en los medios convencionales también llegó a los medios dominantes de la prensa escrita: estudiantes de la Universidad Católica ocuparon dependencias del diario La Tercera (El Ciudadano, 2011).

Medios alternativos. Aquí vale la pena destacar dos experiencias de carácter comunitario-ciudadano, basadas en soportes tradicionales (radio, TV) que, a su vez, tuvieron una articulación online: la Cadena Radial y TV para Chile. Se llamó Cadena Radial a la coproducción de 1800 minutos por la educación pública gratuita y de calidad, realizada entre la Radio Juan Gómez Millas y la Radio Tierra, junto con estudiantes universitarios, otras radios comunitarias y universitarias, medios ciudadanos y centros de producción de Chile y Latinoamérica: 27 en total. Estos 1800 minutos (30 horas) aludían a los U\$1,800 millones que se necesitarían anualmente para educar de forma gratuita a 300 mil estudiantes de Educación Superior. La Cadena tuvo dos versiones en agosto y noviembre de 2011. TV para Chile, por su parte, sale al aire en una primera emisión el 21 de julio de 2011 y una segunda el 30 de agosto como una propuesta en streaming de contenidos con programas en vivo y grabados completando un total de 30 horas o 1800 minutos seguidos (en la misma lógica numérica que la cadena radial), producido por estudiantes de Cine y TV y de Periodismo del Instituto de la Comunicación e Imagen de la Universidad de Chile,

Medios efímeros. En la fase que se inicia en 2011 el movimiento estudiantil se caracteriza por su abundancia de expresiones performativas, entre ellas diversas flashmobs (Thriller por la Educación, suicidios simbólicos masivos, Gagazo por la educación, Superhéroes y villanos por la educación, Besatón por la educación). Alrededor del Palacio de La Moneda también se realizó la protesta llamada "1800 horas por la Educación", donde 
distintas personas sumaron 1800 (horas) corriendo alrededor del Palacio de Gobierno y se hicieron maratones similares en otras ciudades del país como Copiapó, Illapel y Buin.

Web social. El Internet en el movimiento estudiantil estuvo asociado principalmente a su uso como canal de resonancias de la autoexpresión. Las redes sociales (principalmente Twitter y Facebook) fueron usadas, básicamente, para promover, coordinar y difundir formas efímeras de comunicación. Se generaron \# (hashtags) propios del movimiento estudiantil como \#Yoapoyoalosestudiantes, \#estudiantazo y \#movilizados2011; se transmitió vía streaming las marchas o de hitos de esta, como la Toma de la Casa Central de la Universidad de Chile. Estas acciones que generaron una relación de mutua dependencia entre ambas dimensiones comunicativas.

\section{c) Conflictos socio ambientales}

Con respecto a los conflictos socioambientales, se puede considerar como punto de partida del período al movimiento que se revitalizó en 2010 contra la instalación de la termoeléctrica Barracones en Punta de Choros (bahía cercana a La Serena en la IV región), aunque a partir de allí se van levantando en distintas regiones del país donde las demandas territoriales se mezclan con las demandas ambientales.

En cuanto a infraestructuras, el problema más notorio vivido por los conflictos ambientales y territoriales fue el bloqueo de las emisiones por Internet de los medios ciudadanos de la región durante las protestas en Aysén por el alto costo de la vida, durante febrero y marzo de 2012. Esto llevo incluso a una declaración de la organización internacional Reporteros Sin Fronteras (RSF; 2012)

Con respecto a los medios convencionales, la principal denuncia de los movimientos ambientales y territoriales se relaciona con las distintas formas de lavado de imagen por parte de los agentes contaminantes. Destacan sentidos casos como el de los cisnes muertos por la contaminación del río Cruces, la planta de procesamiento de cerdos en Freirina o los efectos contaminantes de la Minera Collahuasi (El Ciudadano, 2010) en el norte de Chile, a 1800 kilómetros de Santiago.

Este lavado de imagen adquiere distintas formas en que se mezclan auspicios, programas especiales hasta llegar a relaciones aún más estrechas, como es el caso del conflicto que sostiene el pueblo de Caimanes contra la contaminación de la Minera Pelambres, propiedad del Grupo económico Luksic (uno de los más importantes del país) y, además, dueño de Canal 13 (uno de los principales canales nacionales de TV abierta) desde 2010.

En relación con medios alternativos, nuevamente las principales expresiones ocurren por medio de las radios; se destacan Santa María, en el caso de Aysén, y Profeta, en el caso de Freirina, ambas con un origen en la Iglesia católica local. La Radio Santa María, si bien con una licencia comercial y bajo administración del arzobispado de la región, realiza un trabajo social importante y que se intensificó en el contexto del conflicto social.

[...] desde que comenzaron las movilizaciones, esta emisora buscó cubrir lo que estaba pasando, enviando desde el primer día un reportero a Puerto Aysén, pero al 
poco tiempo empezaron sus propios auditores a transmitir la necesidad de poder, a través de la radio, contar lo que estaba pasando, sobre todo respecto a las vivencias de represión de las que eran objeto por parte de carabineros y FFEE, frente a lo cual la emisora decidió ajustar la programación para poder canalizar esta demanda de su audiencia y, simultáneamente, amplificar, más allá del cerco informativo circunscrito a la versión oficial, la violencia y la vulneración de derechos que se estaba llevando acabo por parte de FFEE (Moscoso y Munjin, 2013: 29).

Medios efímeros. La principal forma comunicacional de carácter performativo ha sido el surgimiento de diversas asambleas territoriales descentralizadas, por medio de las cuales las diversas comunidades afectadas plantean en lo inmediato sus estrategias para enfrentar a las empresas contaminantes, a las fuerzas policiales, a los representantes del Gobierno y a los medios de comunicación. Pero en una perspectiva más amplia, se están convirtiendo en nuevas formas de distribución del poder político: "Ahora tenemos a lo largo de todo Chile asambleas que actúan con soberanía. Y eso es nuevo. Como lo que ocurrió en Magallanes, Aysén, Calama, Freirina; con Montenegro, Vallenar, Quellón, Chonchi" (El Desconcierto, 2013).

Web social. Un hito en este nivel corresponde a la campaña Chao Pescao / Salvemos Punta de Choros contra la instalación de la termoeléctrica Barracones en una zona de naturaleza casi virgen. La campaña existía al menos desde 2007 y en noviembre del año 2009 se comenzó a viralizar un vídeo contrario a este proyecto medioambiental. La revitalización del conflicto se produjo porque en agosto de 2010 -a pesar de la promesa electoral de Piñera de no aprobar ninguna planta termoeléctrica-, el organismo gubernamental regional encargado (la Corema) aprobó la instalación de la termoeléctrica Barracones en el lugar. Según Olave (2012: 10):

Los seguidores de Chao Pescao en Facebook sumaban 30.740 personas el día anterior a la sesión de la Corema. El día 26 de agosto la suma se encumbró a 43.046 [...] Mientras que en Youtube el día 25 de agosto el documental Salvemos Punta de Choros registró 72.582 reproducciones.

Para esta misma autora, la campaña Chao Pescao puede considerarse una de las primeras movilizaciones ciudadanas realizada primordialmente a través de la web 2.0 en Chile, aunque también con un correlato en manifestaciones callejeras, si bien de mucho menor tamaño. En el caso de las otras movilizaciones territoriales y ambientales, la web social tiende a operar como canal que amplifica lo que se realiza desde los medios alternativos y efímeros.

La siguiente tabla presenta una síntesis de los casos analizados en virtud de las categorías de análisis establecidas 
Tabla 1. Síntesis de hallazgos del análisis de casos4

\begin{tabular}{|c|c|c|c|}
\hline $2010-2013$ & $27 \mathrm{~F}$ y reconstrucción & Movimiento Estudiantil & $\begin{array}{c}\text { Conflictos } \\
\text { socio-ambientales }\end{array}$ \\
\hline $\begin{array}{l}\text { Infraes- } \\
\text { tructuras }\end{array}$ & $\begin{array}{l}\text { Se caen. Fragilidad ante desas- } \\
\text { tres naturales (FS) }\end{array}$ & $\begin{array}{l}\text { Monitoreo de redes sociales } \\
\text { por parte del gobierno (FS) }\end{array}$ & $\begin{array}{l}\text { Hay bloqueo de } \\
\text { conexiones (FS) }\end{array}$ \\
\hline $\begin{array}{l}\text { Medios } \\
\text { convencio- } \\
\text { nales }\end{array}$ & $\begin{array}{l}\text { TV: sensacionalismo respecto } \\
\text { del desastre; omisión de los } \\
\text { movimientos por la reconstruc- } \\
\text { ción (FS) } \\
\text { Mejor evaluación de las radios } \\
\text { nacionales y locales (ambas } \\
\text { comerciales) que de la TV (FS) }\end{array}$ & TV: criminalización (FS) & $\begin{array}{l}\text { TV: criminalización } \\
\text { y/o invisibilización } \\
\text { (FS) }\end{array}$ \\
\hline $\begin{array}{l}\text { Medios al- } \\
\text { ternativos }\end{array}$ & $\begin{array}{l}\text { Radios comunitarias y ciudada- } \\
\text { nas: Noticiero Ciudadano (FP) }\end{array}$ & $\begin{array}{l}\text { Cadena Radial (FP) } \\
\text { TV para Chile (FP) }\end{array}$ & $\begin{array}{l}\text { Radio Santa María } \\
\text { de Aysén / Canal } 40 \\
\text { Aysén (FP) } \\
\text { Radio Profeta (Frei- } \\
\text { rina) (FP) }\end{array}$ \\
\hline $\begin{array}{l}\text { Medios } \\
\text { efímeros }\end{array}$ & $\begin{array}{l}\text { Acciones colectivas de crítica a } \\
\text { la reconstrucción (FS) }\end{array}$ & $\begin{array}{l}\text { Flashmobs de diverso carác- } \\
\text { ter (Thriller por la educación; } \\
\text { Besatón por la educación; } \\
\text { Superhéroes por la educa- } \\
\text { ción y otras) (FP) } \\
\text { Marchas por la educación } \\
\text { (FP) } \\
\text { Acciones colectivas de ase- } \\
\text { dio a los canales de TV (FP) } \\
\text { Acciones individuales (Car- } \\
\text { teles, \#, etc.) (FP) }\end{array}$ & $\begin{array}{l}\text { Surgimiento de } \\
\text { asambleas territo- } \\
\text { riales (FP) }\end{array}$ \\
\hline Web Social & $\begin{array}{l}\text { Canal de autoexpresión y cons- } \\
\text { trucción de relato colectivo } \\
\text { (\#TerremotoChile y otras) (FP) } \\
\text { Voluntariado online que no logra } \\
\text { sustentarse en el tiempo (FP) } \\
\text { Activismo digital por parte de } \\
\text { ONGs en terreno. Competencias } \\
\text { comunicacionales no logran } \\
\text { transferirse a las comunidades } \\
\text { afectadas (FP) }\end{array}$ & $\begin{array}{l}\text { Canal de resonancia de los } \\
\text { medios efímeros (FP) }\end{array}$ & $\begin{array}{l}\text { Canal de resonan- } \\
\text { cia de los medios } \\
\text { efímeros (FP) }\end{array}$ \\
\hline
\end{tabular}

Fuente: elaboración propia

4 La sigla FP se refiere a que la principal fuente de hallazgos en una cuadrícula corresponde a fuentes primarias. La sigla FS se refiere a que la principal fuente de hallazgos en una cuadrícula corresponde a fuentes secundarias. 


\section{Conclusiones}

En primer lugar, observamos la relevancia de visibilizar las características y condicionamientos que suponen las infraestructuras de las redes de telecomunicaciones (acceso) y el control (propiedad) sobre ellas como un aspecto cada vez más relevante para los movimientos sociales en el contexto digital. Si asumimos que ninguna tecnología es neutra (política, económica y socialmente), los hallazgos de nuestros análisis muestran que las brechas en el acceso y apropiación social de los actores sociales movilizados son un llamado a visibilizarlas y tener en consideración que las tecnologías de la información y la comunicación no son autónomas ni transparentes a las relaciones de poder en la sociedad. No todos los actores sociales movilizados en situaciones de crisis pueden acceder de manera equitativa e inclusiva al uso y apropiación estratégica de los servicios asociados a estas redes.

Podemos señalar también que los ciclos de movilizaciones sociales tienen una relación conflictiva o tensión en general con los medios de comunicación convencionales, principalmente con canales de TV abierta nacional y con la prensa escrita de cobertura nacional. Esta relación tiende a ser menos conflictiva con las radios (incluso comerciales), sobre todo si son locales. De manera indirecta, esta tensión es alimentada por el impulso que toma la web social para la expresión de la sociedad moviilizada, donde la comunicación la pueden ejercer directamente con el resto de la ciudadanía sin pasar a través de los medios, lo cual "deja en evidencia una profunda crisis del papel de los intermediarios de la información" (Lara, 2012: 27).

Los medios alternativos juegan un rol importante, aunque generalmente como medios amplificadores de las movilizaciones, antes que como actores que son parte o protagonistas de las mismas. Las excepciones a esta afirmación las encontramos en los casos de Noticiero Ciudadano, Cadena Radial, TV para Chile (las 3 surgidas desde el ICEI) y dos casos en que se trata de radios con un origen religioso, aunque con una orientación al servicio de la comunidad (Aysén y Freirina).

Con respecto a los medios efímeros, notamos que en la medida que son manifestaciones públicas, creativas y masivas, su capacidad de impacto social (llamado de atención, empatía social) está asociada a su alta notoriedad y sentido acumulativo. Por ello la espectacularidad que demostraron en el caso del movimiento estudiantil es más difícil de replicar en el caso de los conflictos socio-ambientales fuera de la capital. Esa puede ser una característica para comprender que la principal expresión performativa en el caso de estos últimos, sea la vuelta a la interacción cara a cara, por medio de las asambleas territoriales.

Ahora bien, un análisis integrado de la movilización social del período muestra que las experiencias mediáticas articuladoras entre los distintos focos de conflicto siguen siendo pocas y no logran consolidarse (aún) en un proyecto mediático de o al servicio de los movimientos sociales, ya sea en forma de plataforma o metodología de trabajo. En 
el caso de los proyectos en soportes digitales, aún no está resuelto plenamente el "para qué", lo cual hace que muchas veces se pierda de vista el sentido de los renovados usos tecnológicos que se hacen de Internet y/o de la telefonía móvil.

Los medios comunitarios y ciudadanos, por ejemplo, se han revitalizado con prácticas de producción de contenido en red, pero eso aún no deriva en que el movimiento se politice o empodere con respecto a su posición dentro del sistema de medios, pese a la coexistencia, durante el mismo período, de diversos movimientos relacionados con la cultura libre en Internet, con una transición a la TV digital más diversa y de mejor calidad, así como con el fortalecimiento de las radios comunitarias, los cuales áun no encuentran eco a sus demandas entre los movimientos sociales cuyos ámbitos de acción son más urgentes y concretos.

El despliegue de los usos auto-expresivos en Internet se produce en un contexto de políticas de comunicación caracterizado por una noción reducida de la libertad de expresión aplicada solo a los profesionales de la información y "un desinterés del Estado por promover el derecho a la comunicación de los sectores marginados, estigmatizados y criminalizados" (Gumucio y Parrini, 2009: 307). Estas políticas también tienen consecuencias en el soporte Internet, donde a pesar del alto número de usuarios de redes sociales y telefonía móvil, ha sido lenta la implementación de la conectividad digital en los sectores rurales y el acceso igualitario a banda ancha (por ejemplo, bajando los precios y garantizando la calidad de la conexión), siguiendo sin ser resueltos los problemas en la infraestructura de telecomunicaciones evidenciadas por el terremoto del 2010. Sin embargo parece existir una brecha entre la propia experiencia comunicacional y el análisis de las condiciones materiales y legales en las cuales se desenvuelven los medios alternativos y que dificultan su sustentabilidad en el tiempo.

En el caso de las radios comunitarias, por ejemplo, en 1994 y 2010 se han establecido los marcos legales dentro de los cuales opera el sector. Aun cuando la Ley de 2010 reconoce débilmente la radiodifusión comunitaria y define lo comunitario, el Estado sigue sin garantizar la libertad de expresión de todos los sectores a través de la radio. La discursividad del poder político sobre la radio comunitaria no se traduce en un real reconocimiento, promoción y fortalecimiento del sector a través de marcos legales que hagan sostenibles estas experiencias sin fines de lucro y gestionadas por la sociedad civil organizada. El sistema de radios chileno sigue concentrado en 4 consorcios que acaparan más del $70 \%$ del sector, mientras las radios comunitarias solo acceden a un $5 \%$ del espectro.

En el caso de la televisión comunitaria, esta no es reconocida legalmente hasta que se introduce en el Congreso la Ley de Televisión Digital. Sin embargo, la ley acaba de ser despachada y no garantiza la sustentabilidad del sector en el entorno digital: no hay un reconocimiento de los canales existentes, no hay apoyos a la transición en términos de infraestructura y solo existen fondos públicos para el financiamiento de contenidos. Todo esto en un contexto de predominio de la televisión comercial, y en donde Chile posee la única televisión pública del mundo que se autofinancia, 
Para nosotros parte de la explicación a esta desacople entre el activismo digital y la demanda por políticas de comunicación más democráticas está relacionada con la estructura de participación ciudadana instaurada en Chile desde el fin de la dictadura y que De la Maza (2005: 61) sintetiza señalando que "las principales políticas sociales implementadas bajo los gobiernos de la Concertación no han tenido entre sus objetivos principales el crear entornos favorables para el fortalecimiento de la sociedad civil", lo cual puede hacerse claramente extensivo a las políticas de comunicación para los medos de la sociedad civil.

Desde la visión que entrega Mata, la pregunta pendiente es cuál es el rol que las organizaciones, agrupaciones, colectivos sociales y ciudadanos le dan a la comunicación en su quehacer y en sus fundamentos, como ciudadanía comunicativa, es decir, "el desarrollo de prácticas tendentes a garantizar los derechos en el campo específico de la comunicación" (Mata, 2006: 13) donde la democratización de las comunicaciones también se reconoce como un campo de cambio y transformación social.

Para cerrar este artículo, desarrollamos una serie de propuestas por donde pensamos que se puede avanzar para mejorar las sinergias entre el activismo digital, la movilización social y las políticas de comunicación:

Preguntarse por el lugar y el sentido estratégico que ocupa la comunicación en las organizaciones chilenas de la sociedad civil. El activismo es fundamentalmente una práctica política y estratégica, pero también supone un accionar comunicacional. Por eso hay que preguntarse por el trabajo comunicacional que piensan, planifican y desarrollan las organizaciones sociales.

Para que la autoexpresión transite hacia la generación de sentidos colectivos no solo importa el uso, per se, de las TIC, sino el sentido del uso y la apropiación estratégica que hacen las organizaciones sociales de las aplicaciones y recursos que ofrece la comunicacional digital; la promesa de participación, colaboración e interconexión, también puede ser un espejismo de participación y finalmente de transformación social

Es importante saber cuál es el nivel de acceso de las organizaciones sociales a Internet. En este proceso, las posibilidades de instalar lógicas y prácticas de activismo a través de las plataformas digitales asume el doble desafío de evitar nuevos procesos de exclusión social, de quienes no están conectados, y a la vez potenciar este espacio como un espacio público, donde articular debates y acciones colectivas.

Tener la claridad y solidez para responder a los para qué y para quienes finalmente se convoca al apoyo y el "me gusta" de los ciudadanos. De manera adicional, hay un desafío importante, cuando ese activismo digital se hace en nombre de "comunidades" que están afectadas por un problema, porque debería incluir un proceso de acompañamiento para que esos otros afectados (un pueblo, un grupo social determinado) también sean capaces de usar y apropiarse de las tecnologías digitales.

Los hallazgos presentados y discutidos en el estudio también llevan a la pregunta (o el desafío) sobre la formación en capacidades o competencias comunicativas digitales de 
las organizaciones, agrupaciones y colectivos de la sociedad civil. Esto no solo pensado desde la lógica de las campañas de alfabetización digital (asociadas a enseñar el uso de un computador e Internet), sino sobre todo para desarrollar creativa y críticamente iniciativas y estrategias que permitan construir comunidades y redes sociales virtuales-reales activas, de sustentabilidad de proyectos comunicativos en el largo plazo, y consolidar finalmente esta sociedad civil que transita entre los territorios reales y los digitales.

\section{Anexo metodológico: fuentes primarias utilizadas}

\section{PROTOCOLO Y MUESTRA DE FUENTES PRIMARIAS DE LA INVESTIGACIÓN}

\section{Entrevistas realizadas a estudiantes coordinadores y/o líderes de los proyectos de comunicación TV para Chile y Cadena Radial}

1.1 Muestra: 6 alumnos participantes en la coordinación general de las 3 primeras emisiones de ambos proyectos (mayo-agosto 2011)

\subsection{Protocolo}

Cómo nació la iniciativa. Eventos clave del proceso. Influencia del proceso de movilizaciones estudiantiles del año 2011

Diagnóstico o reflexión compartida en el equipo que impulsó el proyecto

Identificación de hitos de la experiencia

Autodefinición del proyecto (¿ciudadano?, ¿̇universitario?, ¿̇estudiantil?, ¿̇comunitario?)

Contenidos y tipos de programa emitidos

Organización del trabajo de producción y generación de contenidos

Relación con otras experiencias de movimientos y organizaciones de la sociedad civil y el movimiento educacional

Continuidad y proyección de la experiencia. Metas, objetivos.

Estrategias a desarrollar por los medios ciudadanos, comunitarios, alternativos para potenciar la visibilidad de diversidad social y discursiva

2. Entrevistas realizadas a gestores de radios comunitarias después del terremoto del $\mathbf{2 7}$ de febrero 2010

2.1 Muestra: 20 radios de las regiones más afectadas por el terremoto (Araucanía, Bío-bío, Maule, Bernardo O'higgins y Metropolitana

\subsection{Protocolo}

Equipamiento para situaciones de emergencia

Situación de infraestructura y equipamiento tras terremoto

Reacción o acercamiento de autoridades

Transmisión luego del sismo

Cobertura informativa del terremoto

Carácter o enfoque de la radio

Equipamiento para situaciones de emergencia 
3. Entrevistas a mujeres gestoras de radios comunitarias chilenas, en contexto proceso reconstrucción del terremoto del 27 de febrero de 2010

3.1 Muestra: se identificaron 30 radioemisoras y centros de producción de contenidos. Se seleccionaron las que estuvieran en zonas afectadas por terremoto. Se aplicaron dos cuestionarios a 38 personas en total. La aplicación de este cuestionario se realizó entre junio y agosto de 2011 , en terreno.

A continuación detallamos las dimensiones consideradas en los dos tipos de instrumentos:

\subsubsection{Protocolo dirigido a directores de radios comunitarias $(n=22)$}

Perfil general de director/a de la radio o centro de producción

Perfil general de la radio

Condiciones de transmisión de la radioemisora comunitaria

Programación: cantidad y tipo de programas / en vivo y grabados

Capacidad de producción de contenidos propios

Equipamiento técnico y tecnológico de las radios

Acceso y uso de Internet

Percepción sobre participación de mujeres en las radios

Participación en redes y asociaciones de radios comunitarias (incluyendo AMARC)

\subsubsection{Protocolo dirigido a mujeres que participan en radios comunitarias $(n=16)$}

Perfil general de la radio en la que participa

Perfil de la mujer

Participación de las mujeres en la radio: modalidades y participación en gestión y toma de decisiones

Capacidad de producción de contenidos: prácticas y rutinas

Acceso y uso de Internet

Participación en redes de trabajo de radios comunitarias

\section{Bibliografía}

América Economía (2011, 5 de enero): Explosivo aumento de usuarios de twitter en Chile tras el terremoto. Recuperado el 30 de octubre de 2013. Disponible en: http://tecno.americaeconomia.com/ noticias/explosivo-aumento-de-usuarios-de-twitter-en-chile-tras-el-terremoto

Avendaño, C. (2012) Es la comunicación, estúpido: Empoderamiento comunicacional en las marchas estudiantiles. Ponencia presentada al XI Congreso ALAIC. Montevideo, Recuperado el 25 de octubre de 2013. Disponible en: http://alaic2012.comunicacion.edu.uy/sites/default/files/gt11 _ _ avendaeo _ claudio.pdf

Asociación Chilena de ONGs, ACCiÓN (2011, 17 de junio): Acción presenta reclamo ante el CNTV por nota emitida en Canal 13. Recuperado el 30 de octubre de 2013 Disponible en http://accionag. cl/noticias/accion-presenta-reclamo-ante-el-cntv-por-nota-emitida-en-canal-13/ 
Asociación Mundial de Radios Comunitarias Sección América Latina y Caribe, AMARC ALC (2010): Estado de situación de radios comunitarias en Chile despues del terremoto del 27 de febrero de 2010. Santiago de Chile

Barros, A. (2010): El Comportamiento de la Infraestructura Tecnológica y de Comunicaciones. Cuadernos de Información N. ${ }^{\circ} 26$ enero-junio. Dossier Información y Catástrofes. Pgs. 123 - 137

Cammaerts, B. (2012): Protest Logic and the Mediation Opportunity Structure. European Journal of Communication 27(2). Pgs. 117-134

Castells, M. (1996): La era de la Información. Volumen 1: Economía, Sociedad y Cultural. La Sociedad Red. Madrid: Alianza

Castells, M. (2012): Redes de indignación y esperanza. Madrid: Alianza

Consejo de Ética de los Medios, CEM (2010): Resolución 151 del Consejo de Ética de los Medios Cobertura de noticias en situaciones de catástrofe. Santiago de Chile.

Consejo Nacional de Televisión (2010): Cobertura televisiva del terremoto. La catástrofe vista a través de la pantalla, la audiencia y la industria. Santiago de Chile

Cuello, L. (2011, 14 de julio): Encapuchado de TVN llega al tribunal de ética del Colegio de Periodistas y al CNTV. Recuperado el 30 de octubre de 2013. Disponible es. http://www.otraprensa.com/ encapuchado-de-tvn-llega-al-tribunal-de-etica-del-colegio-de-periodistas-y-al-cntv/

Cuenca, L. (2012): Mapa del despertar comunitario. Los conflictos socioambientales en Chile. En: Mapa de los Conflictos Ambientales. Movilizaciones de Arica a Tierra del Fuego (Pgs. 7 17). Santiago de Chile: Aún creemos en los sueños - Fundación Friedrich Ebert

De la Maza, G. (2005): Tan lejos, tan cerca. Políticas públicas y sociedad civil en Chile. Santiago de Chile: LOM Ediciones.

Downing, J. D. H. (2001): Radical Media: rebellious communication and social movements. California: Thousand Oaks.

Downing, J. D. H. (2008): Social movement theories and alternative media: an evaluation and critique. Communication, Culture and Critique. 1 (1), Pgs. 40-50

Downing, J. D. H. (2010, 2 de julio): ¿Medios libres? De qué y para hacer qué, esas son las preguntas claves. Diagonal N. 129

Downing, J. D. H. (Ed.) (2011a). Encyclopedia of social movement media. London, Thousand Oaks, California, New Delhi, Singapore: Sage.

Downing, J. D. H. (2011b): Medios Radicales, en: J. M. Pereira y A. Cadavid (eds.): Comunicación, Desarrollo y Cambio Social. Interrelaciones entre comunicación, movimientos ciudadanos y medios (Pgs. 399-508). Bogotá: Pontificia Universidad Javeriana-Universidad Minuto de Dios-Unesco

El Ciudadano (2010, 6 de agosto): Minera Collahuasi y su lavado de imagen en Iquique. Recuperado el 30 de octubre de 2013. Disponible en: http://www.elciudadano.cl/2010/06/08/23161/mineracollahuasi-y-su-lavado-de-imagen-en-iquique/

El Ciudadano (2011, 13 de julio): Estudiantes se toman diario La Tercera. Recuperado el 30 de octubre de 2013. Disponible en: http://www.elciudadano.cl/2011/07/13/38385/estudiantes-se-tomandiario-la-tercera/ 
El Desconcierto (2013, 22 de julio): Gabriel Salazar: las asambleas territoriales nos llevan al poder constituyente. Recuperado el 30 de octubre de 201. Disponible en: http://eldesconcierto.cl/ gabriel-salazar-las-asambleas-territoriales-nos-llevan-al-poder-constituyente/

El Mostrador (2011, 30 de julio): Estudiantes se "toman" noticiero de UCV. Recuperado el 30 de octubre de 2013. Disponible en: http://www.elmostrador.cl/varios/2011/06/30/estudiantes-setoman-noticiero-de-ucv/

EMOL (2011, 4 de agosto): Más de 200 estudiantes ingresaron a CHV exigiendo salir al aire. Recuperado el 30 de octubre de 2013. Disponible en: http://www.emol.com/MundoGrafico/index. asp?G_ID=19198\&F_ID=1031394

Gobierno de Chile (2011): Tendencias en redes sociales. Resumen mensual del 13 de junio al 13 de julio. Santiago de Chile

Gumucio, M. y Parrini, V. (2009): Política comunicacional de la concertación. En: Y. Quiroga y J. Ensignia (eds.) Chile en la concertación (1990-2010): una mirada crítica, balance y perspectivas. Tomo 1 (Pp. 303-331). Santiago de Chile: Fundación Friedrich Ebert

Gusfield, J. (1994): La reflexividad de los movimientos sociales: revisión de las teorías sobre la sociedad de masas y el comportamiento colectivo. En: Laraña. E. y Gusfield, J (eds) Los nuevos movimientos sociales: de la ideología a la identidad (Pp. 93-118). Madrid: CIS

Instituto Nacional de Derechos Humanos, INDH (2012): Mapa de conflictos socioambientales en Chile. Santiago de Chile

Lara, T. (2012): ¿Qué caracteriza a la participación ciudadana en la sociedad digital?-En:VV.AA. ParadigmáTIC@s: Comunicación y cultura digital en las ONG de Desarrollo (Pgs. 26-32). Madrid: Coordinadora de ONG para el Desarrollo

Mata, M (2006): Comunicación y ciudadanía: Problemas teórico-políticos de su articulación. Fronteiras - Estudos Midiáticos, VIII (1): 5-15, janeiro/abril.

Moscoso, M. y Munjin, V. (2013): La relación entre movimientos sociales y medios de comunicación en el caso de la radiodifusión en Aysén. Santiago de Chile: PUC-ECO

Miralles, A. M. (2009): Periodismo Público en la Gestión del Riesgo. Lima, Secretaría General de la Comunidad Andina

O'Reilly, T. (2007): What is web 2.0: Design Patterns and Business Models for the Next Generation of Software. Communications \& Strategies, N. ${ }^{\circ} 1$

Olave, A. (2012): La Movilización Ciudadana de Chao Pescao en redes sociales de Internet. El Caso Barracones. Tesis para optar al grado de Magíster en Comunicación Política. Universidad de Chile

Prensa.cl (2011. 5 de julio): La bandera que la TV no mostró ni comentó en el partido de Chile vs México. Recuperado el 30 de octubre de 2013. Disponible en: http://www.prensa.cl/educacion-libre-digna-gratuitabandera-partido-chile-mexico/

Radio BíoBío (2011a, 17 de junio): Acusan que Mineduc dio facilidades a Canal 13 y TVN para mostrar desórdenes en marcha de estudiantes. Recuperado el 30 de octubre de 2013. Disponible en: http://www.biobiochile.cl/2011/06/17/acusan-que-mineduc-dio-facilidades-a-canal-13-y-tvn-paramostrar-desordenes-en-marcha-de-estudiantes.shtml

Radio BíoBío (2011b, 13 de junio): Estudiantes universitarios se 'toman' set de CQC y hacen pasar incómodo momento a sus conductores. Recuperado el 30 de octubre de 2013. Disponible en: 
http://www.biobiochile.cl/2011/06/13/estudiantes-secundarios-se-toman-set-de-cqc-y-hacenpasar-incomodo-momento-a-sus-conductores.shtml

Reporteros Sin Fronteras, RSF (2012, 19 de marzo): Bloqueos de emisiones y represión en Aysén: la libertad de informar dentro del conflicto social. Recuperado el 30 de octubre de 2013. Disponible en: http://es.rsf.org/chile-protestas-en-aysen-y-nuevas-19-03-2012,42157.html

Riechmann, J. y Fernández Buey, F. (1995): Redes que dan Libertad. Introducción a los Nuevos Movimientos Sociales. Barcelona: Paidós.

Rovira, G. (2012): Movimientos sociales, comunicación masiva y comunicación alternativa. Monográfico Portal de la Comunicación INCOM, Universidad Autónoma de Barcelona. Recuperado el 21 de octubre de 2013. Disponible en: http://www.portalcomunicacion.com/monograficos _txt. asp?id=190Etxt=161

Rucht, D. (2004): The Quadruple 'A'. Media Strategies of protest movements since the 1960s. En: Van de Donk, W. E Rucht, D. (eds): Cyber Protest: New media, Citizens, and Social Movements (Pp. 25-48). New York: Routledge

Segura, P. (2012): HidroAysén: el camino sin retorno de la transformación social. En: Mapa de los Conflictos Ambientales. Movilizaciones de Arica a Tierra del Fuego (Pgs. 27 - 32). Santiago de Chile: Aún creemos en los sueños - Fundación Friedrich Ebert

Souza, D. y Martínez, V. (2011): La intervención de la televisión en el terremoto chileno, Comunicar, N. ${ }^{\circ}$ 36 Volumen 18. Pp. 69-76

Sunkel, G. y Geoffroy, E. (2001): Concentración económica de los medios de comunicación. Santiago de Chile: LOM Ediciones

The Lab YER (2010): La explosión en Twitter post-terremoto. Recuperado el 30 de octubre de 2013. Disponible en: http://www.thelabyr.cl/web/wp-content/themes/thelab/pdfs/Explosion _ Twitter_post_terremoto.pdf

Tarrow, S. (2004): El poder en movimiento: los movimientos sociales, la acción colectiva y la política. Madrid: Alianza.

Tilly, C y Wood, L. (2009): Los movimientos sociales 1768 2008: Desde sus orígenes a Facebook. Barcelona: Crítica 